\title{
Initial Boundary Value Problem and Asymptotic Stabilization of the Two-Component Camassa-Holm Equation
}

\author{
Xiju Zong, ${ }^{1}$ Xingong Cheng, ${ }^{1}$ Zhonghua Wang, ${ }^{1}$ and Zhenlai Han ${ }^{2}$ \\ ${ }^{1}$ School of Control Science E Engineering, University of Jinan, Jinan 250022, Shandong, China \\ ${ }^{2}$ School of Science, University of Jinan, Jinan 250022, China \\ Correspondence should be addressed to Xingong Cheng, cse_cxg@ujn.edu.cn \\ Received 9 May 2011; Revised 23 June 2011; Accepted 20 July 2011 \\ Academic Editor: Dirk Aeyels
}

Copyright (C) 2011 Xiju Zong et al. This is an open access article distributed under the Creative Commons Attribution License, which permits unrestricted use, distribution, and reproduction in any medium, provided the original work is properly cited.

The nonhomogeneous initial boundary value problem for the two-component Camassa-Holm equation, which describes a generalized formulation for the shallow water wave equation, on an interval is investigated. A local in time existence theorem and a uniqueness result are achieved. Next by using the fixed-point technique, a result on the global asymptotic stabilization problem by means of a boundary feedback law is considered.

\section{Introduction}

In this paper, we are concerned with the initial boundary value problem and the asymptotic stabilization of the two-component Camassa-Holm equation on a compact interval by means of a stationary feedback law acting on the boundary. The two-component Camassa-Holm equation reads as follows:

$$
\begin{gathered}
u_{t}-u_{x x t}+3 u u_{x}-2 u_{x} u_{x x}-u u_{x x x}+\bar{\rho} \bar{\rho}_{x}=0, \\
\bar{\rho}_{t}+\bar{\rho}_{x} u+\bar{\rho} u_{x}=0,
\end{gathered}
$$

which was first derived as a bi-Hamiltonian models by Olver and Rosenau, see [1]. The system (1.1) shares many features with the Korteweg-De Vries equation Camassa-Holm equation and Degasperis-Procesi Equation; for instance, it has a Lax pair formulation, and it is integrable. In fact, the system (1.1) is related to the first negative flow of the AKNS hierarchy via a reciprocal transformation [2,3]. In [4], Constantin and Ivanov deviated (1.1) 
in the context of shallow water waves theory. As well as they showed that it has global strong solutions and also finite time blow-up solutions. Well-posedness and blow-up results are obtained in $[5,6]$.

For $\bar{\rho} \equiv 0$, the equation (1.1) becomes the Camassa-Holm equation, which is modeling the unidirectional propagation of shallow water waves over a flat bottom. Here $u(t, x)$ stands for the fluid velocity at time $t$ in the spatial $x$ direction [7-11]. The Camassa-Holm equation is also a model for the propagation of axially symmetric waves in hyperelastic rods $[12,13]$. It has a bi-Hamiltonian structure [3] and is completely integrable [7, 14]. Also there is a geometric interpretation of the equation (1.1) in terms of geodesic flow on the diffeomorphism group of the circle $[15,16]$. Its solitary waves are peaked [17]. They are orbitally stable and interact like solitons $[18,19]$.

The Cauchy problem and initial-boundary value problem for the Camassa-Holm equation have been studied extensively in [20-26] and references within. It has been shown that this equation is locally well posed $[20-23,26]$ for some initial data. The advantage of the Camassa-Holm equation in comparison with the KdV equation lies in the fact that the Camassa-Holm equation has peaked solitons and models wave breaking $[27,28]$ (by wave breaking we understand that the wave remains bounded while its slope becomes unbounded in finite time [29]).

For $\bar{\rho} \neq 0$, the Cauchy problems of (1.1) have been discussed in [5, 30], respectively. Recently, a new global existence result and several new blow-up results of strong solutions for the Cauchy problem of (1.1) were obtained in [6]. And a new local existence result and several new blow-up results and blow-up rate of strong solutions for the Cauchy problem of (1.1) defined in a torus were obtained in [31]. Guan and Yin proved the existence of global week solutions to (1.1) provided the initial data satisfying some certain conditions, see [32].

As far as the initial boundary value problem and the asymptotic stabilization of the two-component Camassa-Holm equation on a compact interval are concerned, there are seldom results yet, to the authors' knowledge. Our aim of this paper is to prove the existence of the initial boundary value problem and the asymptotic stabilization of the two-component Camassa-Holm equation on a compact interval by acting on the boundary feedback law, precisely,

(1) the exact controllability problem: given two states $\left(u_{0}, \bar{\rho}_{0}\right)$ and $\left(u_{1}, \bar{\rho}_{1}\right)$ and a time $T>0$, can one find a certain function $v(t)$ such that the solution to (1.1) satisfies $u(T)=u_{1}, \bar{\rho}(T)=\bar{\rho}_{1}$ ? and

(2) the stabilizability problem: can one find a stationary feedback law $v(x)$, such that for any state $\left(u_{0}, \bar{\rho}_{0}\right)$ a solution pair $(u(t), \bar{\rho}(t))$ to closed-loop system is global?

To explain our boundary formulation of (1.1), let us first introduce some transformation, precisely, $m=u-u_{x x}$ and $\rho=\bar{\rho}-1$, which lead the system (1.1) to be equivalent to the system:

$$
\begin{gathered}
m_{t}+u m_{x}+2 m u_{x}+\rho \rho_{x}+\rho_{x}=0, \\
\rho_{t}+u \rho_{x}+\rho u_{x}+u_{x}=0 .
\end{gathered}
$$


Let $T$ be a positive number. In the following we take $\Omega_{T}=[0, T] \times[0,1]$. Let $v_{l}$ and $v_{r}$ be in $C^{0}([0, T], R)$ and $m_{0} \in L^{\infty}(0,1), \rho_{0} \in W^{1, \infty}(0,1)$. We set

$$
\Gamma_{l}=\left\{t \in[0, T] \mid v_{l}(t)>0\right\}, \quad \Gamma_{r}=\left\{t \in[0, T] \mid v_{r}(t)<0\right\} .
$$

In the following, we will always suppose that the sets

$$
P_{l}=\left\{t \in[0, T] \mid v_{l}(t)=0\right\}, \quad P_{r}=\left\{t \in[0, T] \mid v_{r}(t)=0\right\}
$$

have a finite number of connected components. Finally, let $m_{l}, \rho_{l} \in L^{\infty}\left(\Gamma_{l}\right) \times W^{1, \infty}\left(\Gamma_{l}\right)$ and $m_{r}, \rho_{r} \in L^{\infty}\left(\Gamma_{r}\right) \times W^{1, \infty}\left(\Gamma_{r}\right)$. The given functions $v_{l}, v_{r}, m_{l}, \rho_{l}$, and $m_{r}, \rho_{r}$ will be the boundary values for the equation; $m_{0}, \rho_{0}$ are the initial data. Let now $\mathfrak{A}(t, x)$ be the auxiliary function which lifts the boundary values $v_{l}$ and $v_{r}$ and is defined by

$$
\begin{gathered}
\left(1-\partial_{x x}\right) \mathfrak{A}(t, x)=0, \quad \forall(t, x) \in \Omega_{T}, \\
\mathfrak{A}(t, 0)=v_{l}(t), \quad \mathfrak{A}(t, 1)=v_{r}(t), \quad \forall t \in[0, T] .
\end{gathered}
$$

Setting $u=\theta+\mathfrak{A}$, we can further rewrite the system (1.1) as

$$
\begin{gathered}
m(t, x)=\left(1-\partial_{x x}\right) \theta(t, x), \\
\theta(t, 0)=\theta(t, 1)=0, \\
m_{t}+(\theta+\mathfrak{A}) m_{x}=-2 m \partial_{x}(\theta+\mathfrak{A})-\rho \rho_{x}-\rho_{x}, \\
\rho_{t}+(\theta+\mathfrak{A}) \rho_{x}=-(\rho+1) \partial_{x}(\theta+\mathfrak{A}), \\
m(0, \cdot)=m_{0},\left.\quad m(\cdot, 0)\right|_{\Gamma_{l}}=m_{l},\left.\quad m(\cdot, 1)\right|_{\Gamma_{r}}=m_{r}, \\
\rho(0, \cdot)=\rho_{0},\left.\quad \rho(\cdot, 0)\right|_{\Gamma_{l}}=\rho_{l},\left.\quad \rho(\cdot, 1)\right|_{\Gamma_{r}}=\rho_{r} .
\end{gathered}
$$

Let $y=\left(\begin{array}{c}m \\ \rho\end{array}\right), y_{0}=\left(\begin{array}{c}m_{0} \\ \rho_{0}\end{array}\right), b(t, x)=\left(\begin{array}{cc}-2 \partial_{x}(\theta+\mathfrak{A}) & 0 \\ 0 & -\partial_{x}(\theta+\mathfrak{A})\end{array}\right), f(t, x)=\left(\begin{array}{c}-\rho \rho_{x}-\rho_{x} \\ -\partial_{x}(\theta+\mathfrak{A})\end{array}\right)$, and the system (1.7) can be written as

$$
\begin{gathered}
\partial_{t} y+(\theta+\mathfrak{A}) \partial_{x} y=b(t, x) y+f(t, x), \\
y(0, \cdot)=y_{0},\left.\quad y(\cdot, 0)\right|_{\Gamma_{l}}=y_{l},\left.\quad y(\cdot, 1)\right|_{\Gamma_{r}}=y_{r} .
\end{gathered}
$$

We first define what we mean by a weak solution to (1.8). Our test functions will be in the space:

$$
\begin{aligned}
& \operatorname{adm}\left(\Omega_{T}\right)=\left\{\psi \in C^{1}\left(\Omega_{T}\right) \times C^{1}\left(\Omega_{T}\right) \mid \forall x \in[0,1], \psi(t, x)=0 ; \forall t \in[0, T] / \Gamma_{l},\right. \\
& \left.\psi(t, 0)=0 ; \forall t \in[0, T] / \Gamma_{r}, \psi(t, 1)=0\right\} .
\end{aligned}
$$


Definition 1.1. Given $y_{0}=\left(\begin{array}{c}m_{0} \\ \rho_{0}\end{array}\right) \in L^{\infty}\left(\Omega_{T}\right) \times W^{1, \infty}\left(\Omega_{T}\right)$, when $\theta \in L^{\infty}((0, T) ; \operatorname{Lip}([0,1]))$, a function pair $y=\left(\begin{array}{c}m \\ \rho\end{array}\right) \in L^{\infty}\left(\Omega_{T}\right) \times W^{1, \infty}\left(\Omega_{\mathrm{T}}\right)$ is a weak solution to (1.8) if $y$ satisfies

$$
\begin{aligned}
& \iint_{\Omega_{T}}\left[y \partial_{t} \psi+y(\theta+\mathfrak{A}) \partial_{x} \psi-y b(t, x) \psi-f(t, x) \psi\right] \mathrm{d} t \mathrm{~d} x \\
& \quad=-\int_{0}^{1} y_{0} \psi(0, x) \mathrm{d} x+\int_{0}^{T}\left[\psi(t, 1) v_{r}(t) y_{r}(t)-\psi(t, 0) v_{l}(t) y_{l}(t)\right] \mathrm{d} t
\end{aligned}
$$

It is obvious that $C_{0}^{1}\left(\Omega_{T}\right) \times C_{0}^{1}\left(\Omega_{T}\right) \subset \operatorname{adm}\left(\Omega_{T}\right)$; therefore, a weak solution to (1.8) is also a solution to (1.8) in the distribution sense.

Definition 1.2 (see [33]). For $(t, x) \in \Omega_{T}$, let $\varphi(\cdot, t, x)$ be the $C^{1}$ maximal solution to

$$
\begin{gathered}
\partial_{s} \varphi(s, t, x)=a(s, \varphi(s, t, x)), \\
\varphi(t, t, x)=x
\end{gathered}
$$

which is defined on a certain set $[e(t, x), h(t, x)]$ (which is closed because $[0,1]$ is compact) and with possibly $e(t, x)$ and /or $h(t, x)=t$.

We take into account the influence of the boundaries by introducing the sets:

$$
\begin{gathered}
P=\left\{(t, x) \in \Omega_{T} \mid \exists s \in[e(t, x), h(t, x)] \text { such that } \varphi(s, t, x) \in\{0,1\}, a(s, \varphi(s, t, x))=0\right\}, \\
\cup\{(s, \varphi(s, 0,0)) \mid \forall s \in[0, T]\} \cup\{(s, \varphi(s, 0,1)) \mid \forall s \in[0, T]\}, \\
I=\left\{(t, x) \in \Omega_{T} / P \mid e(t, x)=0\right\}, \\
L=\left\{(t, x) \in \Omega_{T} / P \mid \varphi(e(t, x) t, x)=0\right\}, \\
R=\left\{(t, x) \in \Omega_{T} / P \mid \varphi(e(t, x) t, x)=1\right\}, \\
\Gamma_{l}=\{t \in[0, T] \mid a(t, 0)>0\}, \\
\Gamma_{r}=\{t \in[0, T] \mid a(t, 1)>0\} .
\end{gathered}
$$

The following lemma, see [33], will play an important role in proving the local time existence theorem and of a uniqueness result of the initial boundary value problem.

Lemma 1.3. Let $a \in C^{0}\left([0, T] ; C^{1}([0,1])\right), b, f \in L^{\infty}\left(\Omega_{T}\right), y_{0} \in L^{\infty}(0,1), y_{l} \in L^{\infty}\left(\Gamma_{l}\right)$, and $y_{r} \in L^{\infty}\left(\Gamma_{r}\right)$. We will also suppose that the sets:

$$
P_{l}=\left\{t \in[0, T] \mid v_{l}(t)=0\right\}, \quad P_{r}=\left\{t \in[0, T] \mid v_{r}(t)=0\right\}
$$


have at most a countable number of connected components. Then the function $y$, defined by the formula

$$
\begin{aligned}
& \text { for }(t, x) \in P, \quad y(t, x)=0 \text {, } \\
& \text { for }(t, x) \in I, \quad y(t, x)=\exp \left(\int_{0}^{t} b(r, \varphi(r, t, x)) \mathrm{d} r\right) y_{0}(\varphi(0, t, x)) \\
& +\int_{0}^{t} \exp \left(\int_{s}^{t} b(r, \varphi(r, t, x)) \mathrm{d} r\right) f(s, \varphi(s, t, x)) \mathrm{d} s, \\
& \text { for }(t, x) \in L, \quad y(t, x)=\exp \left(\int_{e(t, x)}^{t} b(r, \varphi(r, t, x)) \mathrm{d} r\right) y_{l}(e(t, x)) \\
& +\int_{e(t, x)}^{t} \exp \left(\int_{s}^{t} b(r, \varphi(r, t, x)) \mathrm{d} r\right) f(s, \varphi(s, t, x)) \mathrm{d} s, \\
& \text { for }(t, x) \in R, \quad y(t, x)=\exp \left(\int_{e(t, x)}^{t} b(r, \varphi(r, t, x)) \mathrm{d} r\right) y_{r}(e(t, x)) \\
& +\int_{e(t, x)}^{t} \exp \left(\int_{s}^{t} b(r, \varphi(r, t, x)) \mathrm{d} r\right) f(s, \varphi(s, t, x)) \mathrm{d} s,
\end{aligned}
$$

is a weak solution of

$$
\partial_{t} y+(\theta+\mathfrak{A}) \partial_{x} y=b(t, x) y+f(t, x)
$$

and satisfies

$$
\|y\|_{L^{\infty}\left(\Omega_{T}\right)} \leq\left(\max \left\{\left\|y_{0}\right\|_{L^{\infty}\left(\Omega_{T}\right)},\left\|y_{l}\right\|_{L^{\infty}\left(\Gamma_{l}\right)}\left\|y_{r}\right\|_{L^{\infty}\left(\Gamma_{r}\right)}\right\}+T\|f\|_{L^{\infty}\left(\Omega_{T}\right)}\right) e^{t\|b\|_{L^{\infty}\left(\Omega_{T}\right)}} .
$$

However, if we let $m_{l}, \rho_{l} \in L^{\infty}\left(\Gamma_{l}\right) \times L^{\infty}\left(\Gamma_{l}\right)$ and $m_{r}, \rho_{r} \in L^{\infty}\left(\Gamma_{r}\right) \times L^{\infty}\left(\Gamma_{r}\right)$, note that $f$ depends on the unknown $\rho$ which is not a data; therefore Lemma 1.3 does not hold, or rather Theorem 6 from the Appendix of [33] can not be applied directly to (1.8) (or (1.1)). Indeed if $\theta$ and $\mathfrak{A}$ are given, one can solve the equation on $\rho$ (the equation on the second component in (1.8) (or (1.1))), but this result only guarantee that $\rho$ is in $L^{\infty}$. Therefore the source term in the equation on $m$ is not in $L^{\infty}$ anymore but in $L^{\infty}\left((0, T) ; W^{-1, \infty}(0,1)\right)$, and then Lemma 1.3 cannot be used to solve the transport equation on $m$ (the first component equation on (1.8)). One might try to get more regularity on $\rho$, but in this case more regularity is also needed on $\rho_{0}, \rho_{l}, \rho_{r 0}$ and even on $\theta$ and $\mathfrak{A}$ to get sufficient geometrical assumptions. Then, one might manage $\rho_{0} \in W^{1, \infty}(0,1), \rho_{l} \in W^{1, \infty}\left(\Gamma_{l}\right), \rho_{r} \in W^{1, \infty}\left(\Gamma_{r}\right)$ to obtain at least Lipschitz solution of the scalar transport equation on $\rho$ and then get a weak solution on $m$.

The rest of this paper is organized as follows. In Section 2, the main results of the present paper are stated. Section 3 will be devoted to the proofs of a local time existence theorem and of a uniqueness result of the initial boundary value problem for the system (1.8) (or (1.1)). The problem of asymptotic stabilization for the system is analyzed, and a feedback control law will be investigated in Section 4. 


\section{Main Results}

Theorem 2.1. For $T>0$, we consider $v_{l} \in C^{0}\left(\Gamma_{l}\right), v_{r} \in C^{0}\left(\Gamma_{l}\right)$ such that the sets $P_{l}$ and $P_{r}$ have only a finite number of connected components. Let $y_{0} \in L^{\infty}(0,1) \times W^{1, \infty}(0,1), y_{l} \in L^{\infty}\left(\Gamma_{l}\right) \times W^{1, \infty}\left(\Gamma_{l}\right)$, and $y_{r} \in L^{\infty}\left(\Gamma_{r}\right) \times W^{1, \infty}\left(\Gamma_{r}\right)$. There exist $T>0$ and $(\theta, y)$ a weak solution of the system (1.8) (or (1.1)) with $\theta \in L^{\infty}\left((0, T) ; C^{1,1}([0,1]) \cap \operatorname{Lip}\left((0, T) ; H_{0}^{1}(0,1)\right.\right.$. Moreover any such solution $\theta$ is in fact in $C^{0}\left([0, T] ; W^{2, p}(0,1)\right) \cap C^{1}\left([0, T] ; W_{0}^{1, p}(0,1)\right)$, for all $p<+\infty$. Furthermore the existence time of a maximal solution is larger than $\min \left(\tilde{T}, T^{*}\right)$, with

$$
\begin{gathered}
T^{*}=\max _{\beta>0}\left(\frac{\ln (1+\beta / C)}{2\left[C_{1}+(2+\sinh (1))(\beta+C)\right]}\right), \\
C=\max \left\{\left\|y_{0}\right\|_{L^{\infty} \times W^{1, \infty}},\left\|y_{l}\right\|_{L^{\infty} \times W^{1, \infty}},\left\|y_{r}\right\|_{L^{\infty} \times W^{1, \infty}}\right\}, \\
C_{1}=\frac{1}{\tanh (1)}\left(\left\|v_{l}\right\|_{L^{\infty}(0, T)},\left\|v_{r}\right\|_{L^{\infty}(0, T)}\right) .
\end{gathered}
$$

In a second step, we will show a weak-strong uniqueness property.

Theorem 2.2. Let $\theta \in L^{\infty}\left((0, T) ; C^{1,1}([0,1]) \cap \operatorname{Lip}\left((0, T) ; H_{0}^{1}(0,1)\right.\right.$, and let $y \in \operatorname{Lip}([0,1]) \times$ $\operatorname{Lip}([0,1])$ be a weak solution of (1.8) (or (1.1)); then it is unique in the function space $\left[L^{\infty}\left((0, T) ; C^{1,1}([0,1]) \times L^{\infty}\left(\Omega_{T}\right)\right)\right]^{2}$.

Let $A_{l}>2 \sinh (1), A_{r}>A_{l} \cosh (1)+\sinh (2), T>0$ and $M$ a symmetric matrix, and assume that $\rho_{0}, \rho_{l}$, and $\rho_{r}$ have compact supports in $(0,1) / \Gamma_{l} / \Gamma_{r}$, respectively. Our feedback law for (1.8) (or (1.1)) reads

$$
y \in C^{0}([0,1]) \times C^{0}([0,1]) \longmapsto\left\{\begin{array}{l}
v_{l}(y)=A_{l}\|y\|_{C^{0}([0,1]) \times C^{0}([0,1])^{\prime}} \\
v_{r}(y)=A_{r}\|y\|_{C^{0}([0,1]) \times C^{0}([0,1])^{\prime}} \\
\dot{y}_{l}(t)=M y_{l}(t) .
\end{array}\right.
$$

Theorem 2.3. For any $y_{0} \in\left[C^{0}([0,1])\right]^{2}$ there exists $(y, v) \in\left[C^{0}\left(\Omega_{T}\right)\right]^{2} \times C^{2}([0,1])$ a weak solution of (1.1) and (2.2) satisfying

$$
\forall x \in[0,1], \quad y(0, x)=y_{0}(x)
$$

Furthermore any maximal solution of (1.1), (2.2), and (2.3) is global, and if we let

$$
c=\min \left\{A_{l}-2 \sinh (1), \frac{A_{r}-A_{l} \cosh (1)+\sinh (2)}{\sinh (1)}\right\}, \quad \tau=\frac{1}{\|M\|_{2}} \ln \left(\frac{c\|y\|_{C^{0}([0,1])}}{\|M\|_{2}}\right),
$$


then we have

$$
\|y\|_{C^{0}([0,1])} \leq \frac{\|M\|_{2}}{c\left[1+\|M\|_{2}(t-\tau)\right]} .
$$

Remark 2.4. For $\rho \equiv 0$, the system (1.1) becomes the classical Camassa-Holm equation, and the above theorems degenerate those of [33] with $k=0$.

\section{Proofs of the Main Theorems}

\subsection{Local Existence Theorem}

This strategy is borrowed from [33]. We want to solve (1.6) (1.8) (or (1.1)). Equation (1.6) is a linear elliptic equation, and with $\theta$ fixed (1.8) is a linear transport equation in $y$, with boundary data.

Given $\theta \in L^{\infty}\left((0, T) ; C^{1,1}([0,1])\right) \cap \operatorname{Lip}\left((0, T) ; H_{0}^{1}(0,1)\right)$, we will define $y=\left(\begin{array}{c}m \\ \rho\end{array}\right)$ to be the solution to (1.8), and once we have $m$ in $L^{\infty}\left(\Omega_{T}\right)$, we introduce $\tilde{\theta}$ solution of

$$
\left(1-\partial_{x x}\right) \tilde{\theta}=m
$$

Then $\mp$ is defined as the operator $\tilde{\theta}=\mathscr{F}(\theta)$

Lemma 3.1. The function $\mathfrak{A}$ defined by (1.5) satisfies

$$
\begin{aligned}
\forall(t, x) \in \Omega_{T}, \quad \mathfrak{A}(t, x) \in C^{0}\left([0, T] ; C^{\infty}([0,1])\right), \\
\mathfrak{A}(t, x)=\frac{1}{\sinh (1)}\left(\sinh (x) \boldsymbol{v}_{r}(t)+\sinh (1-x) v_{l}(t)\right), \\
\quad\|\mathfrak{A}(t, x)\|_{L^{\infty}\left((0, T) ; C^{1,1}([0,1])\right)} \leq \frac{\cosh (1)}{\sinh (1)}\left(\left\|v_{l}\right\|_{L^{\infty}(0, T)}+\left\|v_{r}\right\|_{L^{\infty}(0, T)}\right) .
\end{aligned}
$$

Then for a function $\theta \in L^{\infty}\left((0, T) ; C^{1,1}([0,1])\right) \cap \operatorname{Lip}\left((0, T) ; H_{0}^{1}(0,1)\right)$, we consider $\varphi$ the flow of $\theta+\mathfrak{A}$.

Lemma 3.2. The flow $\varphi$ satisfies the following properties.

(1) $\varphi$ is $C^{1}$ with the following partial derivatives:

$$
\begin{aligned}
& \partial_{1} \varphi(s, t, x)=(\theta+\mathfrak{A})(s, \varphi(s, t, x)), \\
& \partial_{2} \varphi(s, t, x)=-(\theta+\mathfrak{A}) \exp \left(\int_{t}^{s} \partial_{x}(\theta+\mathfrak{A})(r, \varphi(r, t, x)) \mathrm{d} r\right), \\
& \partial_{3} \varphi(s, t, x)=\exp \left(\int_{t}^{s} \partial_{x}(\theta+\mathfrak{A})(r, \varphi(r, t, x)) \mathrm{d} r\right) .
\end{aligned}
$$


(2) For all $j=1,2,3$,

$$
\left\|\partial_{j} \varphi\right\|_{C^{0}} \leq\left(1+\|\theta+\mathfrak{A}\|_{C^{0}\left(\Omega_{T}\right)}\right) e^{T\|\theta+\mathfrak{A}\|_{C^{0}\left(\Omega_{T}\right)}}
$$

For $(t, x) \in \Omega_{T}, \varphi(\cdot, t, x)$ is defined on a set $[e(t, x), h(t, x)]$, here $e(t, x)$ is basically the entrance time in $\Omega_{T}$ of the characteristic curve going through $(t, x)$.

(3) If $e(t, x)>0$, then $\varphi(e(t, x), t, x) \in\{0,1\}$.

(4) If $h(t, x)<T$, then $\varphi(h(t, x), t, x) \in\{0,1\}$.

For $\theta \in L^{\infty}\left((0, T) ; C^{1,1}([0,1])\right) \cap \operatorname{Lip}\left((0, T) ; H_{0}^{1}(0,1)\right)$, we define the solution to (1.8) $y \in L^{\infty}\left(\Omega_{T}\right) \times W^{1, \infty}\left(\Omega_{T}\right)$ by

$$
\begin{aligned}
& \text { for }(t, x) \in I, \quad y(t, x)=\left(\begin{array}{cc}
\exp \int_{0}^{t} E_{1} \mathrm{~d} r & 0 \\
0 & \exp \int_{0}^{t} E_{2} \mathrm{~d} r
\end{array}\right) y_{0}(\varphi(0, t, x)) \\
& +\int_{0}^{t}\left(\begin{array}{cc}
\exp \int_{s}^{t} E_{1} \mathrm{~d} r & 0 \\
0 & \exp \int_{s}^{t} E_{2} \mathrm{~d} r
\end{array}\right)\left(\begin{array}{c}
-\rho \rho_{x}-\rho_{x} \\
-\partial_{x}(\theta+\mathfrak{A})
\end{array}\right) \mathrm{d} s, \\
& \text { for }(t, x) \in L, \quad y(t, x)=\left(\begin{array}{cc}
\exp \int_{e(t, x)}^{t} E_{1} \mathrm{~d} r & 0 \\
0 & \exp \int_{e(t, x)}^{t} E_{2} \mathrm{~d} r
\end{array}\right) y_{l}(e(t, x)) \\
& \begin{array}{r}
+\int_{e(t, x)}^{t}\left(\begin{array}{cc}
\exp \int_{s}^{t} E_{1} \mathrm{~d} r & 0 \\
0 & \exp \int_{s}^{t} E_{2} \mathrm{~d} r
\end{array}\right)\left(\begin{array}{c}
-\rho \rho_{x}-\rho_{x} \\
-\partial_{x}(\theta+\mathfrak{A})
\end{array}\right) \mathrm{d} s, \\
\text { for }(t, x) \in R, \quad y(t, x)=\left(\begin{array}{cc}
\exp \int_{e(t, x)}^{t} E_{1} \mathrm{~d} r & 0 \\
0 & \exp \int_{e(t, x)}^{t} E_{2} \mathrm{~d} r
\end{array}\right) y_{r}(e(t, x))
\end{array} \\
& \begin{array}{c}
+\int_{e(t, x)}^{t}\left(\begin{array}{cc}
\exp \int_{s}^{t} E_{1} \mathrm{~d} r & 0 \\
0 & \exp \int_{s}^{t} E_{2} \mathrm{~d} r
\end{array}\right)\left(\begin{array}{c}
-\rho \rho_{x}-\rho_{x} \\
-\partial_{x}(\theta+\mathfrak{A})
\end{array}\right) \mathrm{d} s, \\
\text { for }(t, x) \in R, \quad y(t, x)=\left(\begin{array}{cc}
\exp \int_{e(t, x)}^{t} E_{1} \mathrm{~d} r & 0 \\
0 & \exp \int_{e(t, x)}^{t} E_{2} \mathrm{~d} r
\end{array}\right) y_{r}(e(t, x))
\end{array} \\
& +\int_{e(t, x)}^{t}\left(\begin{array}{cc}
\exp \int_{s}^{t} E_{1} \mathrm{~d} r & 0 \\
0 & \exp \int_{s}^{t} E_{2} \mathrm{~d} r
\end{array}\right)\left(\begin{array}{c}
-\rho \rho_{x}-\rho_{x} \\
-\partial_{x}(\theta+\mathfrak{A})
\end{array}\right) \mathrm{d} s
\end{aligned}
$$


with

$$
E_{1}=-2 \partial_{x}(\theta+\mathfrak{A})(r, \varphi(r, t, x)), \quad E_{2}=-\partial_{x}(\theta+\mathfrak{A})(r, \varphi(r, t, x)) .
$$

And we have

(1) the function $y$ is the unique weak solution of (1.8) in the sense of Definition 4.1; thanks to Lemma 1.3. $y$ is in $L^{\infty} \cap W^{1, \infty}$.

Remark 3.3. $y$ is the only weak solution of (1.8), and also $y$ is in $L^{\infty} \cap W^{1, \infty}$ which is crucial for the stabilization problem because of the coupling between the two components of $y$. However, rather thanks to the regularity on the boundary data $\rho$ is indeed Lipschitz inside the zones $L, R$, and $I$; it ensures that the transition between those zones should be continuous under the kind of compatibility conditions between $\rho_{0}, \rho_{l}$, and $\rho_{r}$; for example, all three have a compact support in $(0,1) / \Gamma_{l} / \Gamma_{r}$.

(2) Since $y \in L^{\infty}\left(\Omega_{T}\right) \times W^{1, \infty}\left(\Omega_{T}\right)$, we immediately get $y \in W^{1, \infty}\left(0, T ; H^{-1}(0,1)\right) \times$ $W^{2, \infty}\left(0, T ; H^{-1}(0,1)\right)$ and satisfies (1.8). Also we can get the estimates:

$$
\begin{aligned}
\|y(t, x)\|_{L^{\infty}\left(\Omega_{T}\right) \times L^{\infty}\left(\Omega_{T}\right)} \leq & C_{0} \exp \left[2 T\left(\left\|\partial_{x} \theta\right\|_{L^{\infty}\left(\Omega_{T}\right)}+\left\|\partial_{x} \mathfrak{A}\right\|_{L^{\infty}\left(\Omega_{T}\right)}\right)\right], \\
\left\|\partial_{t} y(t, x)\right\|_{L^{\infty}\left(0, T ; H^{-1}\right) \times L^{\infty}\left(0, T ; H^{-1}\right)} \leq & 3 C_{0} \exp \left[2 T\left(\left\|\partial_{x} \theta\right\|_{L^{\infty}\left(\Omega_{T}\right)}+\left\|\partial_{x} \mathfrak{A}\right\|_{L^{\infty}\left(\Omega_{T}\right)}\right)\right], \\
& \times\left(\|\theta\|_{L^{\infty}((0, T) ; \operatorname{Lip}([0,1]))}+\|\mathfrak{A}\|_{L^{\infty}((0, T) ; \operatorname{Lip}([0,1]))}\right),
\end{aligned}
$$

where

$$
\begin{aligned}
C_{0}= & \max \left\{\left\|y_{0}\right\|_{L^{\infty} \times W^{1, \infty \infty}},\left\|y_{l}\right\|_{L^{\infty} \times W^{1, \infty}},\left\|y_{r}\right\|_{L^{\infty} \times W^{1, \infty}}\right\} \\
& +T\left[\left(\|\rho\|_{L^{\infty}}+1\right)\left\|\rho_{x}\right\|_{L^{\infty}((0, T) ; \operatorname{Lip}([0,1]))}+\left\|\partial_{x} \theta\right\|_{L^{\infty}\left(\Omega_{T}\right)}+\left\|\partial_{x} \mathfrak{A}\right\|_{L^{\infty}\left(\Omega_{T}\right)}\right] .
\end{aligned}
$$

(3) If $(t, x) \in I \cup L \cup R$ and if $\left(s, s^{\prime}\right) \in[e(t, x), h(t, x)]^{2}$, one has the following property:

$$
y(s, \varphi(s, t, x))=\left(\begin{array}{cc}
\exp \int_{0}^{t} E_{1} \mathrm{~d} r & 0 \\
0 & \exp \int_{0}^{t} E_{2} \mathrm{~d} r
\end{array}\right) y\left(s^{\prime}, \varphi\left(s^{\prime}, t, x\right)\right) .
$$

From the elliptic equation we can get

Lemma 3.4. There exists a unique $\tilde{\theta} \in L^{\infty}\left((0, T) ; H_{0}^{1}(0,1)\right)$ such that

$$
\left(1-\partial_{x x}\right) \tilde{\theta}=m
$$


holds in $D^{\prime}(0,1)$ for all $(t, x) \in \Omega_{T}$. Furthermore $\tilde{\theta} \in L^{\infty}\left((0, T) ; C^{1,1}([0,1])\right) \cap \operatorname{Lip}\left([0, T] ; H_{0}^{1}(0,1)\right)$, since $m \in L^{\infty}\left(\Omega_{T}\right) \cap \operatorname{Lip}\left([0, T] ; H^{-1}(0,1)\right)$. Moreover we have the bounds:

$$
\begin{gathered}
\|\tilde{\theta}\|_{L^{\infty}\left((0, T) ; C^{1,1}([0,1])\right)} \leq[1+\sinh (1)]\|m\|_{L^{\infty}\left(\Omega_{T}\right)} \leq[1+\sinh (1)]\|y\|_{L^{\infty}\left(\Omega_{T}\right) \times W^{1, \infty}\left(\Omega_{T}\right)^{\prime}} \\
\left\|\partial_{t} \tilde{\theta}\right\|_{L^{\infty}\left((0, T) ; H_{0}^{1}(0,1)\right)} \leq\left\|\partial_{t} m\right\|_{L^{\infty}\left((0, T) ; H^{-1}\right)} \leq\left\|\partial_{t} y\right\|_{L^{\infty}\left((0, T) ; H^{-1}\right) \times W^{1, \infty}\left((0, T) ; H^{-1}\right)} .
\end{gathered}
$$

The proof can be found in [33] and omitted.

Thus, for $\theta \in L^{\infty}\left((0, T) ; C^{1,1}(0,1)\right) \cap \operatorname{Lip}\left([0, T] ; H_{0}^{1}(0,1)\right)$, the operator $\mp$ can be defined as $\tilde{\theta}=\mathcal{F}(\theta) \in L^{\infty}\left((0, T) ; C^{1,1}(0,1)\right) \cap \operatorname{Lip}\left([0, T] ; H_{0}^{1}(0,1)\right)$.

Let $B_{0}$ and $B_{1}$ be positive numbers, then we set

$$
\begin{gathered}
\mathcal{C}_{B_{0}, B_{1}, T}=\left\{\theta \in L^{\infty}\left((0, T) ; C^{1,1}(0,1)\right) \cap \operatorname{Lip}\left([0, T] ; H_{0}^{1}(0,1)\right) \mid \text { both }\|\theta\|_{L^{\infty}\left((0, T) ; C^{1,1}(0,1)\right)} \leq B_{0}\right. \\
\left.\|\theta\|_{\operatorname{Lip}\left([0, T] ; H^{-1}(0,1)\right)} \leq B_{1}\right\} .
\end{gathered}
$$

Obviously $\mathcal{C}_{B_{0}, B_{1}, T}$ is convex. We will endow $\mathcal{C}_{B_{0}, B_{1}, T}$ with the norm $\|\theta\|_{L^{\infty}([0, T] ; \operatorname{Lip}(0,1))}$.

Lemma 3.5. There exist positive numbers $B_{0}, B_{1}$ and $T$, such that $F$ maps $\mathcal{C}_{B_{0}, B_{1}, T}$ into itself.

Proof. The proceeding of proof is similar to that of [33, Lemma 3], but the constant $C_{0}$ differs slightly from that of [33, Lemma 3]. Let us first introduce the two following constants:

$$
\begin{aligned}
C_{0}= & \max \left\{\left\|y_{0}\right\|_{L^{\infty} \times W^{1, \infty}},\left\|y_{l}\right\|_{L^{\infty} \times W^{1, \infty}},\left\|y_{r}\right\|_{L^{\infty} \times W^{1, \infty}}\right\} \\
& +T\left[\left(\|\rho\|_{L^{\infty}}+1\right)\left\|\rho_{x}\right\|_{L^{\infty}((0, T) ; \operatorname{Lip}([0,1]))}+\left\|\partial_{x} \theta\right\|_{L^{\infty}\left(\Omega_{T}\right)}+\left\|\partial_{x} \mathfrak{A}\right\|_{L^{\infty}\left(\Omega_{T}\right)}\right], \\
C_{1}= & \frac{1}{\tanh (1)}\left(\left\|v_{l}\right\|_{L^{\infty}(0, T)},\left\|v_{r}\right\|_{L^{\infty}(0, T)}\right) .
\end{aligned}
$$

Estimates (3.7), and (3.11) on $y$ and $\theta$ now read,

$$
\begin{aligned}
\|y(t, x)\|_{L^{\infty}\left(\Omega_{T}\right) \times W^{1, \infty}\left(\Omega_{T}\right)} \leq & C_{0} \exp \left[2 T\left(\left\|\partial_{x} \theta\right\|_{L^{\infty}\left(\Omega_{T}\right)}+C_{1}\right)\right], \\
\left\|\partial_{t} y(t, x)\right\|_{L^{\infty}\left((0, T) ; H^{-1}\right) \times L^{\infty}\left((0, T) ; H^{-1}\right)} \leq & 3 C_{0} \exp \left[2 T\left(\left\|\partial_{x} \theta\right\|_{L^{\infty}\left(\Omega_{T}\right)}+C_{1}\right)\right] \\
& \times\left(\|\theta\|_{L^{\infty}((0, T) ; \operatorname{Lip}([0,1]))}+C_{1}\right), \\
\|\tilde{\theta}\|_{L^{\infty}\left((0, T) ; C^{1,1}([0,1])\right)} \leq & {[1+\sinh (1)]\|y(t, x)\|_{L^{\infty}\left(\Omega_{T}\right) \times W^{1, \infty}\left(\Omega_{T}\right)^{\prime}} } \\
\left\|\partial_{t} \tilde{\theta}\right\|_{L^{\infty}\left((0, T) ; H_{0}^{1}(0,1)\right)} \leq & \left\|\partial_{t} y(t, x)\right\|_{L^{\infty}\left((0, T) ; H^{-1}\right) \times L^{\infty}\left((0, T) ; H^{-1}\right)} .
\end{aligned}
$$


Combining those estimates we get for all $\theta \in \mathcal{C}_{B_{0}, B_{1}, T}$ :

$$
\begin{aligned}
\|\tilde{\theta}\|_{L^{\infty}\left((0, T) ; C^{1,1}([0,1])\right)} & \leq C_{0}[1+\sinh (1)] \exp \left[2 T\left(\left\|\partial_{x} \theta\right\|_{L^{\infty}\left(\Omega_{T}\right)}+C_{1}\right)\right] \\
& \leq C_{0}[1+\sinh (1)] \exp \left[2 T\left(B_{0}+C_{1}\right)\right], \\
\left\|\partial_{t} \tilde{\theta}\right\|_{L^{\infty}\left((0, T) ; H_{0}^{1}(0,1)\right)} & \leq 3 C_{0} \exp \left[2 T\left(\left\|\partial_{x} \theta\right\|_{L^{\infty}\left(\Omega_{T}\right)}+C_{1}\right)\right]\left(\|\theta\|_{L^{\infty}((0, T) ; \operatorname{Lip}([0,1]))}+C_{1}\right) \\
& \leq 3 C_{0} \exp \left[2 T\left(B_{0}+C_{1}\right)\right]\left(B_{0}+C_{1}\right) .
\end{aligned}
$$

To obtain $\tilde{\theta} \in \mathcal{C}_{B_{0}, B_{1}, T}$, it is sufficient that

$$
\begin{gathered}
C_{0}[1+\sinh (1)] \exp \left[2 T\left(B_{0}+C_{1}\right)\right] \leq B_{0}, \\
B_{0}+3 C_{0} \exp \left[2 T\left(B_{0}+C_{1}\right)\right]\left(B_{0}+C_{1}\right) \leq B_{1} .
\end{gathered}
$$

Once we have chosen $T$ and $B_{0}$, it is easy to choose $B_{1}$ to satisfy the second inequality. For the first one we just choose $B_{0}$ sufficiently large and then $T$ close to 0 . More precisely,

$$
\begin{gathered}
C=\max \left\{\left\|y_{0}\right\|_{L^{\infty} \times W^{1, \infty}},\left\|y_{l}\right\|_{L^{\infty} \times W^{1, \infty},}\left\|y_{r}\right\|_{L^{\infty} \times W^{1, \infty}}\right\}, \\
B_{0}>C[1+\sinh (1)], \quad T \leq \frac{\ln B_{0} /(C[1+\sinh (1)])}{2\left(B_{0}+C_{1}\right)},
\end{gathered}
$$

we complete the proof by taking $B_{0} /(C[1+\sinh (1)])=C+\beta, \beta>0$.

Lemma 3.6. $\mathcal{C}_{B_{0}, B_{1}, T}$ is compact with respect to the norm $\|\cdot\|_{L^{\infty}([0, T] ; \operatorname{Lip}(0,1))}$.

The proof is very similar to that appeared in [33] and omitted.

Lemma 3.7. For $y \in H^{s} \times H^{s-1}, s>2, f(y)$ is bounded on bounded sets in $H^{s} \times H^{s-1}$. Therefore, $f(y)$ is bounded on bounded sets in $L^{\infty} \times L^{\infty}$ by the embedding theorem.

The proof is very similar to that appeared in [33] and omitted.

Lemma 3.8. The operator $\mathcal{F}: \mathcal{C}_{B_{0}, B_{1}, T} \rightarrow \mathcal{C}_{B_{0}, B_{1}, T}$ is continuous with respect to the norm $\|\theta\|_{L^{\infty}([0, T] ; \operatorname{Lip}(0,1))}$.

Proof. Take a sequence $\left\{\theta_{n}\right\}$ which tends to $\theta$ with respect to $\|\cdot\|_{L^{\infty}([0, T] ; \operatorname{Lip}(0,1))}$, set $\tilde{\theta}_{n}=\mp \theta_{n}$ and $\tilde{\theta}=\mp \theta$, denote by $\varphi_{n}$ the flow of $\theta_{n}+\mathfrak{A}$ and $\varphi$ the flow of $\theta+\mathfrak{A}$, and we have that $\varphi_{n} \rightarrow \varphi$ locally in $C^{1}$ as $n \rightarrow \infty$, thanks to Proposition A.4 in [33]. What we will need to do is to show that $m_{n} \rightarrow m$ in $L^{1}(0,1)$ as $n \rightarrow \infty$ and $\rho_{n} \rightarrow \rho$ in $L^{1}(0,1)$ as $n \rightarrow \infty$.

Let $t \in[0, T]$, having supposed that $P_{l}$ and $P_{r}$ have only a finite number of connected components, we can assume, reducing $t$ if necessary that $v_{l}$ and $v_{r}$ do not change sign on $[0, t]$. Since the characteristics of $\varphi_{n}$ and $\varphi$ may or may not cross before time $t$, we only consider the case that $\varphi(t, 0,0) \leq \varphi_{n}(t, 0,0) \leq \varphi(t, 0,1) \leq \varphi_{n}(t, 0,1)$, without loss of generality. The other 
cases are proved in the same way. We first point out that since $\theta_{n} \in \mathcal{C}_{B_{0}, B_{1}, T}$ we have a bound for $\left\{y_{n}\right\}$ in $L^{\infty}\left(\Omega_{T}\right)$. Now

$$
\begin{aligned}
& \int_{0}^{1}\left|y(t, x)-y_{n}(t, x)\right| \mathrm{d} x \\
& \quad=\left(\int_{0}^{\varphi(t, 0,0)}+\int_{\varphi(t, 0,0)}^{\varphi_{n}(t, 0,0)}+\int_{\varphi_{n}(t, 0,0)}^{\varphi(t, 0,1)}+\int_{\varphi(t, 0,1)}^{\varphi_{n}(t, 0,1)}+\int_{\varphi_{n}(t, 0,1)}^{1}\right)\left|y(t, x)-y_{n}(t, x)\right| \mathrm{d} x \\
& \quad=I_{1}+I_{2}+I_{3}+I_{4}+I_{5} .
\end{aligned}
$$

Since $\varphi_{n}(t, 0,0) \rightarrow \varphi(t, 0,0)$ as $n \rightarrow \infty$ and $\varphi_{n}(t, 0,1) \rightarrow \varphi(t, 0,1)$ as $n \rightarrow \infty$ and thanks to the uniform bound on $\left\|y_{n}\right\|_{L^{\infty}}$, we see that both $I_{2}$ and $I_{4}$ tend to 0 when $n$ goes to infinity.

For $I_{1}$ we have

$$
\begin{aligned}
& I_{1}=\int_{0}^{\varphi(t, 0,0)}\left|y(t, x)-y_{n}(t, x)\right| \mathrm{d} x \\
& =\int_{0}^{\varphi(t, 0,0)} \mid\left(\begin{array}{cc}
\exp \int_{e(t, x)}^{t} E_{1} \mathrm{~d} r & 0 \\
0 & \exp \int_{e(t, x)}^{t} E_{2} \mathrm{~d} r
\end{array}\right) y_{l}(e(t, x)) \\
& +\int_{e(t, x)}^{t}\left(\begin{array}{cc}
\exp \int_{s}^{t} E_{1} \mathrm{~d} r & 0 \\
0 & \exp \int_{s}^{t} E_{2} \mathrm{~d} r
\end{array}\right)\left(\begin{array}{c}
-\rho \rho_{x}-\rho_{x} \\
-\partial_{x}(\theta+\mathfrak{A})
\end{array}\right) \mathrm{d} s \\
& -\left(\begin{array}{cc}
\exp \int_{e_{n}(t, x)}^{t} E_{1 n} \mathrm{~d} r & 0 \\
0 & \exp \int_{e_{n}(t, x)}^{t} E_{2 n} \mathrm{~d} r
\end{array}\right) y_{l}\left(e_{n}(t, x)\right) \\
& -\int_{e_{n}(t, x)}^{t}\left(\begin{array}{cc}
\exp \int_{s}^{t} E_{1 n} \mathrm{~d} r & 0 \\
0 & \exp \int_{s}^{t} E_{2 n} \mathrm{~d} r
\end{array}\right)\left(\begin{array}{c}
-\rho \rho_{x}-\rho_{x} \\
-\partial_{x}\left(\theta_{n}+\mathfrak{A}\right)
\end{array}\right) \mathrm{d} s \mid \mathrm{d} x,
\end{aligned}
$$

where

$$
E_{1 n}=-2 \partial_{x}\left(\theta_{n}+\mathfrak{A}\right)\left(r, \varphi_{n}(r, t, x)\right), \quad E_{2}=-\partial_{x}\left(\theta_{n}+\mathfrak{A}\right)\left(r, \varphi_{n}(r, t, x)\right) .
$$

Thanks to the boundedness on the $\|f\|_{L^{\infty} \times L^{\infty}}$ (Lemma 3.7) and Proposition A.2 of [33], if $(t, x) \bar{\epsilon} P$ we have $\varphi_{n}(t, 0,0) \rightarrow \varphi(t, 0,0)$ as $n \rightarrow \infty$. This implies that if $y_{l}$ was continuous, since we have a uniform bound on $\left\|\theta_{n}\right\|_{L^{\infty}\left((0, T) ; C^{1,1}(0,1)\right)}$ the dominated convergence theorem would provide: $I_{1} \rightarrow 0 . I_{3} \rightarrow 0$ and $I_{5} \rightarrow 0$ which can be obtained by using the same method. Therefore, for $y_{0}, y_{l}$ and $y_{r}$ continuous we have $\left\|y(t, \cdot)-y_{n}(t, \cdot)\right\|_{L^{1}} \rightarrow 0$. 
From the inequality (56) in [33], we obtain

$$
\begin{aligned}
\|y(t, \cdot)\|_{L^{1}(0,1) \times L^{1}(0,1)} \leq & {\left[\left\|y_{0}\right\|_{L^{1}(0,1) \times L^{1}(0,1)}+\left\|y_{l}\right\|_{L^{1}(0, t) \cap \Gamma_{l} \times L^{1}(0, t) \cap \Gamma_{l}}\right.} \\
& +\left\|y_{r}\right\|_{L^{1}(0, t) \cap \Gamma_{r} \times L^{1}(0, t) \cap \Gamma_{r}}+\left(\|\rho\|_{L^{1}(0,1) \times L^{1}(0,1)}+1\right)\left\|\rho_{x}\right\|_{L^{1}(0,1) \times L^{1}(0,1)} \\
& \left.+\left\|\partial_{x}(\theta+\mathfrak{A})\right\|_{L^{1}(0,1)}\right]\left\|\partial_{x}(\theta+\mathfrak{A})\right\|_{L^{\infty}\left(\Omega_{T}\right)} \exp \left(3 t\left\|\partial_{x}(\theta+\mathfrak{A})\right\|_{L^{\infty}\left(\Omega_{T}\right)}\right), \\
\left\|y_{n}(t, \cdot)\right\|_{L^{1}(0,1) \times L^{1}(0,1)} \leq[ & \left\|y_{0}\right\|_{L^{1}(0,1) \times L^{1}(0,1)}+\left\|y_{l}\right\|_{L^{1}(0, t) \cap \Gamma_{l} \times L^{1}(0, t) \cap \Gamma_{l}} \\
& +\left\|y_{r}\right\|_{L^{1}(0, t) \cap \Gamma_{r} \times L^{1}(0, t) \cap \Gamma_{r}}+\left(\left\|\rho_{n}\right\|_{L^{1}(0,1) \times L^{1}(0,1)}+1\right)\left\|\rho_{n, x}\right\|_{L^{1}(0,1) \times L^{1}(0,1)} \\
& \left.+\left\|\partial_{x}\left(\theta_{n}+\mathfrak{A}\right)\right\|_{L^{1}(0,1)}\right]\left\|\partial_{x}\left(\theta_{n}+\mathfrak{A}\right)\right\|_{L^{\infty}\left(\Omega_{T}\right)} \exp \left(3 t\left\|\partial_{x}\left(\theta_{n}+\mathfrak{A}\right)\right\|_{L^{\infty}\left(\Omega_{T}\right)}\right) .
\end{aligned}
$$

So the general case of convergence $\left\|y(t, \cdot)-y_{n}(t, \cdot)\right\|_{L^{1}} \rightarrow 0$ follows from the density of $C^{0}$ in $L^{1}$ and the uniform bound on $\left\|\theta_{n}\right\|_{L^{\infty}((0, T) ; \operatorname{Lip}(0,1))}$.

Now only the restriction on $t$ remains; we recall that until now we supposed that $v_{l}$ and $v_{r}$ did not change sign on $[0, t]$. If $v_{l}$ and $v_{r}$ do not change sign on $\left[0, t_{1}\right]$ and then on $\left[t_{1}, t\right]$, we have

$$
\left\|y\left(t_{1}, \cdot\right)-y_{n}\left(t_{1}, \cdot\right)\right\|_{L^{1}} \longrightarrow 0
$$

Let $\tilde{y}_{n}$ the solution of

$$
\begin{gathered}
\partial_{t} \tilde{y}_{n}+\left(\theta_{n}+\mathfrak{A}\right) \partial_{x} \tilde{y}_{n}=b_{n}(t, x) \tilde{y}_{n}+f_{n}(t, x), \\
\tilde{y}_{n}\left(t_{1}, \cdot\right)=y_{t_{1}},\left.\quad \tilde{y}_{n}(\cdot, 0)\right|_{\Gamma_{l}}=y_{l},\left.\quad \tilde{y}_{n}(\cdot, 1)\right|_{\Gamma_{r}}=y_{r} .
\end{gathered}
$$

We can conclude that as $n \rightarrow \infty$,

$$
\begin{aligned}
\| y(t, \cdot)- & y_{n}(t, \cdot) \|_{L^{1}(0,1) \times L^{1}(0,1)} \\
\leq & \left\|y(t, \cdot)-\tilde{y}_{n}(t, \cdot)\right\|_{L^{1}(0,1) \times L^{1}(0,1)}+\left\|\tilde{y}_{n}(t, \cdot)-y_{n}(t, \cdot)\right\|_{L^{1}(0,1) \times L^{1}(0,1)} \\
\leq & \left\|y\left(t_{1}, \cdot\right)-\tilde{y}_{n}\left(t_{1}, \cdot\right)\right\|_{L^{1}(0,1) \times L^{1}(0,1)} \exp \left(3\left(t-t_{1}\right)\left\|\partial_{x}\left(\theta_{n}+\mathfrak{A}\right)\right\|_{L^{\infty}\left(\Omega_{T}\right)}\right) \\
& +\left\|y(t, \cdot)-\tilde{y}_{n}(t, \cdot)\right\|_{L^{1}(0,1) \times L^{1}(0,1)} \\
\leq & \left\|y\left(t_{1}, \cdot\right)-y_{n}\left(t_{1}, \cdot\right)\right\|_{L^{1}(0,1) \times L^{1}(0,1)} \exp \left(3\left(t-t_{1}\right)\left\|\partial_{x}\left(\theta_{n}+\mathfrak{A}\right)\right\|_{L^{\infty}\left(\Omega_{T}\right)}\right) \\
& +\left\|y(t, \cdot)-\tilde{y}_{n}(t, \cdot)\right\|_{L^{1}(0,1) \times L^{1}(0,1)} \longrightarrow 0 .
\end{aligned}
$$


Thus the convergence in $L^{1}(0,1)$ propagates on each interval where $v_{l}$ and $v_{r}$ do not change sign; thanks to the hypothesis on $P_{r}$ and $P_{l}$ we have that for all $t \in[0, T]$, $\left\|y(t, \cdot)-y_{n}(t, \cdot)\right\|_{L^{1}(0,1)} \rightarrow 0$, as $n \rightarrow \infty$. Combining this first convergence result with the uniform bound and using the dominated convergence theorem in the time variable, we obtain $\left\|y-y_{n}\right\|_{L^{1}\left(\Omega_{T}\right)} \rightarrow 0$ which implies that

$$
\left\|\tilde{\theta}-\tilde{\theta}_{n}\right\|_{L^{1}\left(0, T ; W^{2,1}(0,1)\right)} \longrightarrow 0 .
$$

From the compactness of $\mathcal{C}_{B_{0}, B_{1}, T}$, we get that $\widetilde{\theta}_{n} \rightarrow \tilde{\theta}$ holds in $\mathcal{C}_{B_{0}, B_{1}, T}$.

All the above lemmas result in the application of Schauders fixed point theorem to $F$ and we get a solution

$$
\theta \in L^{\infty}\left((0, T) ; C^{1,1}(0,1)\right) \cap \operatorname{Lip}\left([0, T] ; H_{0}^{1}(0,1)\right)
$$

From the construction of F and from Proposition A.8 in [33] the additional regularity properties of any solution $\theta$,

$$
\theta \in C^{0}\left([0, T] ; W^{2, p}(0,1)\right) \cap C^{1}\left([0, T] ; W_{0}^{1, p}(0,1)\right), \quad \forall p<+\infty .
$$

\subsection{Uniqueness}

In this subsection, we will show that the solution to the system (1.6) and (1.8) is unique; that to say, given $(y, \theta)$ and $(\tilde{y}, \tilde{\theta})$ be two solutions of (1.6) and (1.8) for the same initial and boundary data, we will get $y=\tilde{y}$ and $\theta=\tilde{\theta}$.

Let $Y=y-\tilde{y}, \Theta=\theta-\widetilde{\theta}, B(t, x)=b(t, x)-\tilde{b}(t, x), F(t, x)=f(t, x)-\tilde{f}(t, x)$, then $\Theta \in \operatorname{Lip}\left([0, T] ; H_{0}^{1}(0,1)\right)$. And we have $M=m-\tilde{m} \in L^{\infty}\left(\Omega_{T}\right)$ and $Y \in L^{\infty}\left(\Omega_{T}\right) \times L^{\infty}\left(\Omega_{T}\right)$ is the solution to

$$
\begin{gathered}
\partial_{t} Y+(\theta+\mathfrak{A}) \partial_{x} Y=b Y+B(t, x) \tilde{y}-\Theta \partial_{x} \tilde{y}+F(t, x) \\
Y(0, \cdot)=0,\left.\quad Y(\cdot, 0)\right|_{\Gamma_{l}}=0,\left.\quad Y(\cdot, 1)\right|_{\Gamma_{r}}=0
\end{gathered}
$$

Using the lemma again with $b(t, x)=\left(\begin{array}{cc}-2 \partial_{x}(\theta+\mathfrak{A}) & 0 \\ 0 & -\partial_{x}(\theta+\mathfrak{A})\end{array}\right)$ and $\bar{f}=B(t, x) \tilde{y}-U \partial_{x} \tilde{y}+F(t, x)$, we get

$$
\begin{aligned}
& \text { for }(t, x) \in P, \quad Y(t, x)=0 \\
& \text { for }(t, x) \in I, \quad Y(t, x)=\int_{0}^{t} \exp \left(\int_{s}^{t} b(r, \varphi(r, t, x)) \mathrm{d} r\right) \bar{f}(s, \varphi(s, t, x)) \mathrm{d} s,
\end{aligned}
$$




$$
\begin{aligned}
& \text { for }(t, x) \in L, \quad Y(t, x)=\int_{e(t, x)}^{t} \exp \left(\int_{s}^{t} b(r, \varphi(r, t, x)) \mathrm{d} r\right) \bar{f}(s, \varphi(s, t, x)) \mathrm{d} s, \\
& \text { for }(t, x) \in R, \quad Y(t, x)=\int_{e(t, x)}^{t} \exp \left(\int_{s}^{t} b(r, \varphi(r, t, x)) \mathrm{d} r\right) \bar{f}(s, \varphi(s, t, x)) \mathrm{d} s .
\end{aligned}
$$

Since $\|U(t, \cdot)\|_{L^{\infty}(0,1)} \leq C^{\prime}\|Y(t, \cdot)\|_{L^{\infty} \times L^{\infty}}$ for some positive constant $C^{\prime}$, and $y, \partial_{x} y$ bounded, we see that for some $C^{\prime \prime}>0$,

$$
\|\bar{f}(t, \cdot)\|_{L^{\infty} \times L^{\infty}} \leq C^{\prime \prime}\|Y(t, \cdot)\|_{L^{\infty} \times L^{\infty}} .
$$

And since $b(t, x)$ is bounded, we get that

$$
\|Y(t, \cdot)\|_{L^{\infty} \times L^{\infty}} \leq C^{\prime \prime} \int_{0}^{s}\|Y(s, \cdot)\|_{L^{\infty} \times L^{\infty}} \mathrm{d} s .
$$

Then we complete the proof of the uniqueness by using Gronwall's lemma.

\section{Stabilization}

\subsection{Preliminary Results}

The equilibrium state that we want to stabilize is $y=0, \theta=\mathfrak{A}=0$. A natural idea is using Lyapunov indirection method to investigate whether the linearized system around the equilibrium state is stabilizable or not. Its stabilization would provide a local stabilization result on the nonlinear system. Unfortunately, the linearized system is not stabilizable, for the state of the linearized system around the equilibrium state is constant. We see that the sign of $\theta+\mathfrak{A}$ controls the geometry of the characteristics, and the sign of $\partial_{x}(\theta+\mathfrak{A})$ controls the ingredient information of $y$ along the characteristics. We will use the return method that Coron introduced in [34]. We would like our feedback law, $v_{l}(y)=A_{l}\|y\|_{C^{0}([0,1]) \times C^{0}([0,1])}$ $v_{r}(y)=A_{r}\|y\|_{C^{0}([0,1]) \times C^{0}([0,1])}$, to provide $\theta+\mathfrak{A} \geq 0$ and $\partial_{x}(\theta+\mathfrak{A}) \geq 0$. However, there is a difficulty in the stabilization problem. It needs not to be true that the transition between those zones is continuous rather thanks to the regularity on the boundary data $\rho$ is indeed Lipschitz inside the zones $L, R$, and $I$. To achieve this target, we have to prescribe $y_{l}$, and we just need to make a continuous transition at $(t, x)=(0,0)$ and let $y_{l}$ asymptotically converge in time; we assume that compatibility conditions hold; precisely, $\rho_{0}, \rho_{l}$ and $\rho_{r}$ have compact supports in $(0,1) / \Gamma_{l} / \Gamma_{r}$, respectively. To achieve this target, we have to prescribe $y_{l}$, and we just need to make a continuous transition at $(t, x)=(0,0)$ and let $y_{l}$ asymptotically converge in time. This is guaranteed by

$$
\partial_{t} y_{l}=M y_{l}
$$

where $M$, symmetric matrix, is the unique matrix solution to the matrix function:

$$
P M+M^{T} P=-Q,
$$


for some symmetric positive-definite matrices, $P$ and $Q$. Indeed, let $V\left(t, y_{l}\right)=y_{l}^{T} P y_{l}$ be the Lyapunov candidate, and that $y_{l}$ asymptotically converges in time is equivalent to that the time derivative of the $V, \dot{V}=y_{l}^{T}\left(P M+M^{T} P\right) y_{l}$ is strictly negative. A fixed-point strategy will be used again to prove the existence of a solution to the closed-loop system. We begin by defining the domain of the operator.

Definition 4.1. Let $X$ be the space of $(g, N) \in C^{0}([0, T] ;[0,1])^{2} \times C^{0}([0, T])$ satisfying

(1) $g(0, x)=y_{0}(x), g(t, 0)=y_{0}(0) e^{M t}$,

(2) $\|g(t, \cdot)\|_{C^{0}([0,1])} \leq N(t)$,

(3) $N(t)$ is nonincreasing and $N(0) \leq\left\|y_{0}\right\|_{C^{0}([0,1]) \times C^{0}([0,1])}$.

Lemma 4.2. The domain $X$ is nonempty, convex, bounded, and closed with respect to the uniform topology.

Taking $M=\left(\begin{array}{c}\lambda, 0 \\ 0, \lambda\end{array}\right), \lambda<0$, satisfying (4.1) and (4.2), and $\left(y_{0}(x) e^{M t},\left\|y_{0}\right\|_{C^{0}([0,1])^{2}} e^{M t}\right) \in$ $X$, so $X$ is nonempty.

Now for $(y, N(t)) \in X$ we define $\breve{\theta}$ and $\breve{\mathfrak{A}}$ as the solutions of

$$
\begin{gathered}
m(t, x)=\left(1-\partial_{x x}\right) \breve{\theta}(t, x), \\
\breve{u}(t, 0)=\breve{\theta}(t, 1)=0, \quad \forall(t, x) \in \Omega_{T}, \\
\left(1-\partial_{x x}\right) \breve{\mathfrak{A}}(t, x)=0, \\
\breve{\mathfrak{A}}(t, 0)=A_{l} N(t), \quad \breve{\mathfrak{A}}(t, 1)=A_{r} N(t), \quad \forall(t, x) \in \Omega_{T} .
\end{gathered}
$$

That is,

$$
\begin{aligned}
\forall(t, x) \in \Omega_{T}, \quad \breve{u}(t, x) & =-\int_{0}^{x} \sinh (x-\xi) m(t, \xi) \mathrm{d} \xi, \\
\breve{\mathfrak{A}}(t, x) & =\frac{N(t)}{\sinh (1)}\left[\sinh (x) A_{r}+\sinh (1-x) A_{l}\right] .
\end{aligned}
$$

Thus, we have the estimates:

$$
\begin{gathered}
|\breve{\theta}(t, x)| \leq 2 \sinh (1)\|y\|_{C^{0}([0,1]) \times C^{0}([0,1])^{\prime}} \\
\left|\partial_{x} \breve{\theta}(t, x)\right| \leq 2 \cosh (1)\|y\|_{C^{0}([0,1]) \times C^{0}([0,1])^{\prime}} \\
\left|\partial_{x x} \breve{\theta}(t, x)\right| \leq[1+2 \sinh (1)]\|y\|_{C^{0}([0,1]) \times C^{0}([0,1])^{\prime}} \\
\left|\partial_{x} \breve{\mathfrak{A}}(t, x)\right| \geq \frac{A_{r}-2 \cosh (1)}{\sinh (1)} N(t), \\
|\breve{\mathfrak{A}}(t, x)| \geq A_{l} N(t) .
\end{gathered}
$$


And in turn,

$$
\begin{gathered}
(\breve{\theta}+\breve{\mathfrak{A}})(t, x) \geq\left[A_{l}-2 \sinh (1)\right]\|y\|_{C^{0}([0,1]) \times C^{0}([0,1])^{\prime}} \\
\partial_{x}(\breve{\theta}+\breve{\mathfrak{A}})(t, x) \geq \frac{A_{r}-2 \cosh (1) A_{l}-\sinh (2)}{\sinh (1)}\|y\|_{C^{0}([0,1]) \times C^{0}([0,1])} .
\end{gathered}
$$

Now, if $\varphi$ is the flow of $\breve{\theta}+\breve{\mathfrak{A}}, e$ is $C^{1}$ and since $\breve{u}+\breve{\mathfrak{A}} \geq 0, \varphi(\cdot, t, x)$ is nondecreasing. Thus we can define the entrance time and then the operator $\mathcal{S}$ as follows. with

$$
\text { Let } e(t, x)=\min \{s \in[0, T] \mid \varphi(s, t, x)=0\}, \text { for }(t, x) \in[0, T] \times[0,1], \mathcal{S}(y, N)=(\widetilde{y}, \widetilde{N})
$$

(1) if $x \geq \varphi(t, 0,0)$,

$$
\begin{aligned}
\tilde{y}(t, x)= & \left(\begin{array}{cc}
\exp \int_{0}^{t} E_{1} \mathrm{~d} r & 0 \\
0 & \exp \int_{0}^{t} E_{2} \mathrm{~d} r
\end{array}\right) y_{0}(\varphi(0, t, x)) \\
& +\int_{0}^{t}\left(\begin{array}{cc}
\exp \int_{s}^{t} E_{1} \mathrm{~d} r & 0 \\
0 & \exp \int_{s}^{t} E_{2} \mathrm{~d} r
\end{array}\right)\left(\begin{array}{c}
-\rho \rho_{x}-\rho_{x} \\
-\partial_{x}(\theta+\mathfrak{A})
\end{array}\right) \mathrm{d} s,
\end{aligned}
$$

(2) if $x \leq \varphi(t, 0,0)$,

$$
\begin{aligned}
\tilde{y}(t, x)= & \left(\begin{array}{cc}
\exp \int_{0}^{t} E_{1} \mathrm{~d} r & 0 \\
0 & \exp \int_{0}^{t} E_{2} \mathrm{~d} r
\end{array}\right) e^{M e(t, x)} y_{0}(0) \\
& +\int_{e(t, x)}^{t}\left(\begin{array}{cc}
\exp \int_{s}^{t} E_{1} \mathrm{~d} r & 0 \\
0 & \exp \int_{s}^{t} E_{2} \mathrm{~d} r
\end{array}\right)\left(\begin{array}{c}
-\rho \rho_{x}-\rho_{x} \\
-\partial_{x}(\theta+\mathfrak{A})
\end{array}\right) \mathrm{d} s,
\end{aligned}
$$

(3) $N(t)=\|y(t, \cdot)\|_{C^{0}([0,1]) \times C^{0}([0,1])}$.

From Lemma 1.3 we know that $\tilde{y}$ is the weak solution of

$$
\begin{gathered}
\partial_{t} \tilde{y}+(\theta+\mathfrak{A}) \partial_{x} \tilde{y}=b(t, x) \tilde{y}+f(t, x), \\
\tilde{y}(0, \cdot)=0, \quad \tilde{y}(t, 0)=e^{M t} y_{0}(0) .
\end{gathered}
$$


Lemma 4.3. (1) The operator $\mathcal{S}$ maps $X$ to $X$.

(2) The family $\mathcal{S}(X)$ is uniformly bounded and equicontinuous.

(3) $S$ is continuous with respect to the uniform topology.

The proof is very similar to [33] except for here the state $y$ is a two-component vector and the proof is omitted.

We can apply Schauder's fixed-point theorem to $S$ and get $(y, N)$ fixed point of $S$.

\subsection{Stabilization and Global Existence}

From (4.6) and (4.7), for all $(t, x) \in \Omega_{T}$,

$$
\begin{gathered}
(\breve{\theta}+\breve{\mathfrak{A}})(t, x) \geq c\|y(r, \cdot)\|_{C^{0}([0,1]) \times C^{0}([0,1])^{\prime}} \\
\partial_{x}(\breve{\theta}+\breve{\mathfrak{A}})(t, x) \geq c\|y(r, \cdot)\|_{C^{0}([0,1]) \times C^{0}([0,1])^{\prime}}
\end{gathered}
$$

$y$ is the solution of the transport equation (1.8) and it satisfies

$$
\begin{aligned}
y(t, x)= & \left(\begin{array}{cc}
\exp \int_{s}^{t} E_{1} \mathrm{~d} r^{\prime} & 0 \\
0 & \exp \int_{s}^{t} E_{2} \mathrm{~d} r
\end{array}\right) y(s, \varphi(s, t, x)) \\
& +\int_{s}^{t}\left(\begin{array}{cc}
\exp \int_{r}^{t} E_{1} \mathrm{~d} r & 0 \\
0 & \exp \int_{r}^{t} E_{2} \mathrm{~d} r^{\prime}
\end{array}\right) f(r, x) \mathrm{d} r .
\end{aligned}
$$

We get for $t \geq s$

$$
|y(t, x)| \leq \exp \left(-\int_{s}^{t} c\|y(r, \cdot)\|_{C^{0}([0,1]) \times C^{0}([0,1])} \mathrm{d} r\right) y|s, \varphi(s, t, x)| .
$$

This implies that $|y(t, x)|=\sqrt{|m(t, x)|^{2}+|\rho(t, x)|^{2}}$ decreases along the characteristics (strictly for the times where $y(t, \cdot) \neq 0$ ). But we have also imposed $y(t, 0)=y(s, 0) e^{M(t-s)}$; therefore $|y(t, x)|$ also decreases along $x=0$. This already shows, thanks to the existence theorem, that a maximal solution of the closed loop system is global. To get a more precise statement, we consider all the characteristics between time $t$ and $s$, and we obtain

$$
\begin{aligned}
\|y(t, \cdot)\|_{C^{0}([0,1]) \times C^{0}([0,1])} \leq & \max _{r \in[s, t]}\left\{e^{\|M\|_{2}(s-r)} \exp \left(-\int_{s}^{t} c\|y(r, \cdot)\|_{C^{0}([0,1]) \times C^{0}([0,1])} \mathrm{d} r\right)\right\} \\
& \times\|y(t, \cdot)\|_{C^{0}([0,1]) \times C^{0}([0,1])^{\prime}}
\end{aligned}
$$


Now we define

$$
g(r)=e^{\|M\|_{2}(s-r)} \exp \left(-\int_{s}^{t} c\|y(r, \cdot)\|_{C^{0}([0,1]) \times C^{0}([0,1])} \mathrm{d} r\right)
$$

Then

$$
g^{\prime}(r)=\left[c\|y(t, \cdot)\|_{C^{0}([0,1]) \times C^{0}([0,1])}-\|M\|_{2}\right] g(r),
$$

and we know that as long as the quantity $\|y(t, \cdot)\|_{C^{0}([0,1]) \times C^{0}([0,1])}$ is not equal to zero, it strictly decreases. So if $\left\|y_{0}\right\|_{C^{0}([0,1]) \times C^{0}([0,1])}>\|M\|_{2} / c$, for $t$ small enough $\|y(t, \cdot)\|_{C^{0}([0,1]) \times C^{0}([0,1])} \geq$ $\|M\|_{2} / c$, and we have

$$
\|y(t, \cdot)\|_{C^{0}([0,1]) \times C^{0}([0,1])} \leq e^{-\|M\|_{2} t}\left\|y_{0}\right\|_{C^{0}([0,1]) \times C^{0}([0,1])^{\prime}}
$$

which implies $\|y(\tau, \cdot)\|_{C^{0}([0,1]) \times C^{0}([0,1])} \leq\|M\|_{2} / c$. This provides for $\tau \leq s \leq t$, the inequality

$$
\|y(t, \cdot)\|_{C^{0}([0,1]) \times C^{0}([0,1])} \leq e^{\left.-\int_{s}^{t} c \| y(r,)\right) \|_{C^{0}([0,1]) \times 0^{0}([0,1)} \mathrm{d} r}\|y(s, \cdot)\|_{C^{0}([0,1]) \times C^{0}([0,1])} .
$$

And we conclude with a classical comparison principle for ODES.

\section{Acknowledgments}

The authors would like to express their gratitude to the reviewer and editor for the valuable comments and suggestions. The first author would like to express his gratitude to Professor Adrain Constantin for leading his attention to this new system and Professor Zhaoyang Yin for suggesting the present problem. This work was supported by the National Natural Scientific Foundation of China (Grants 11026112 and 61074021). This work is partially supported by the Shandong Province Young and Middle aged Scientists Research Award Fund (Grant no. BS2011SF001).

\section{References}

[1] P. J. Olver and P. Rosenau, "Tri-Hamiltonian duality between solitons and solitary-wave solutions having compact support," Physical Review E, vol. 53, no. 2, pp. 1900-1906, 1996.

[2] M. Chen, S.-Q. Liu, and Y. Zhang, "A two-component generalization of the Camassa-Holm equation and its solutions," Letters in Mathematical Physics, vol. 75, no. 1, pp. 1-15, 2006.

[3] G. Falqui, "On a Camassa-Holm type equation with two dependent variables," Journal of Physics A, vol. 39, no. 2, pp. 327-342, 2006.

[4] A. Constantin and R. I. Ivanov, "On an integrable two-component Camassa-Holm shallow water system," Physics Letters. A, vol. 372, no. 48, pp. 7129-7132, 2008.

[5] J. Escher, O. Lechtenfeld, and Z. Yin, "Well-posedness and blow-up phenomena for the 2-component Camassa-Holm equation," Discrete and Continuous Dynamical Systems A, vol. 19, no. 3, pp. 493-513, 2007.

[6] C. Guan and Z. Yin, "Global existence and blow-up phenomena for an integrable two-component Camassa-Holm shallow water system," Journal of Differential Equations, vol. 248, no. 8, pp. 2003-2014, 2010. 
[7] R. Camassa and D. D. Holm, "An integrable shallow water equation with peaked solitons," Physical Review Letters, vol. 71, no. 11, pp. 1661-1664, 1993.

[8] A. Constantin and D. Lannes, "The hydrodynamical relevance of the Camassa-Holm and DegasperisProcesi equations," Archive for Rational Mechanics and Analysis, vol. 192, no. 1, pp. 165-186, 2009.

[9] D. Ionescu-Kruse, "Variational derivation of the Camassa-Holm shallow water equation," Journal of Nonlinear Mathematical Physics, vol. 14, no. 3, pp. 303-312, 2007.

[10] R. I. Ivanov, "Water waves and integrability," Philosophical Transactions of the Royal Society of London A, vol. 365, no. 1858, pp. 2267-2280, 2007.

[11] R. S. Johnson, "Camassa-Holm, Korteweg-de Vries and related models for water waves," Journal of Fluid Mechanics, vol. 457, pp. 63-82, 2002.

[12] A. Constantin and W. Strauss, "Stability of a class of solitary waves in compressible elastic rods," Physics Letters. A, vol. 270, no. 3-4, pp. 140-148, 2000.

[13] H.-H. Dai, "Model equations for nonlinear dispersive waves in a compressible Mooney-Rivlin rod," Acta Mechanica, vol. 127, no. 1-4, pp. 193-207, 1998.

[14] A. Constantin, "On the scattering problem for the Camassa-Holm equation," Proceedings of The Royal Society of London A, vol. 457, no. 2008, pp. 953-970, 2001.

[15] A. Constantin and B. Kolev, "Geodesic flow on the diffeomorphism group of the circle," Commentarii Mathematici Helvetici, vol. 78, no. 4, pp. 787-804, 2003.

[16] B. Kolev, "Bi-Hamiltonian systems on the dual of the Lie algebra of vector fields of the circle and periodic shallow water equations," Philosophical Transactions of the Royal Society of London A, vol. 365, no. 1858, pp. 2333-2357, 2007.

[17] R. Camassa, D. D. Holm, and J. M. Hyman, "A new integrable shallow water equation," Advances in Applied Mechanics, vol. 31, pp. 1-33, 1994.

[18] R. Beals, D. H. Sattinger, and J. Szmigielski, "Multi-peakons and a theorem of Stieltjes," Inverse Problems, vol. 15, no. 1, pp. L1-L4, 1999.

[19] A. Constantin and W. A. Strauss, "Stability of peakons," Communications on Pure and Applied Mathematics, vol. 53, no. 5, pp. 603-610, 2000.

[20] A. Constantin and J. Escher, "Global existence and blow-up for a shallow water equation," Annali della Scuola Normale Superiore di Pisa, vol. 26, no. 2, pp. 303-328, 1998.

[21] A. Constantin and J. Escher, "Well-posedness, global existence, and blowup phenomena for a periodic quasi-linear hyperbolic equation," Communications on Pure and Applied Mathematics, vol. 51, no. 5, pp. 475-504, 1998.

[22] R. Danchin, "A few remarks on the Camassa-Holm equation," Differential and Integral Equations, vol. 14, no. 8, pp. 953-988, 2001.

[23] M. Lakshmanan, "Integrable nonlinear wave equations and possible connections to tsunami dynamics," in Tsunami and Nonlinear Waves, pp. 31-49, Springer, Berlin, Germany, 2007.

[24] J. Escher and Z. Yin, "Initial boundary value problems of the Camassa-Holm equation," Communications in Partial Differential Equations, vol. 33, no. 1-3, pp. 377-395, 2008.

[25] J. Escher and Z. Yin, "Initial boundary value problems for nonlinear dispersive wave equations," Journal of Functional Analysis, vol. 256, no. 2, pp. 479-508, 2009.

[26] G. Rodríguez-Blanco, "On the Cauchy problem for the Camassa-Holm equation," Nonlinear Analysis, vol. 46, no. 3, pp. 309-327, 2001.

[27] R. Camassa, D. D. Holm, and J. M. Hyman, "A new integrable shallow water equation," Advances in Applied Mechanics, vol. 31, pp. 1-33, 1994.

[28] A. Constantin and J. Escher, "Wave breaking for nonlinear nonlocal shallow water equations," Acta Mathematica, vol. 181, no. 2, pp. 229-243, 1998.

[29] G. B. Whitham, Linear and Nonlinear Waves, John Wiley \& Sons, New York, NY, USA, 1980.

[30] A. Constantin and R. I. Ivanov, "On an integrable two-component Camassa-Holm shallow water system," Physics Letters. A, vol. 372, no. 48, pp. 7129-7132, 2008.

[31] Q. Hu and Z. Yin, "Well-posedness and blow-up phenomena for a periodic 2-component CamassaHolm equation," Proceedings of the Royal Society of Edinburgh, vol. 140A, pp. 1-15, 2010.

[32] C. Guan and Z. Yin, "Global weak solutions for a two-component Camassa-Holm shallow water system," Journal of Functional Analysis, vol. 260, no. 4, pp. 1132-1154, 2011.

[33] V. Perrollaz, "Initial boundary value problem and asymptotic stabilization of the Camassa-Holm equation on an interval," Journal of Functional Analysis, vol. 259, no. 9, pp. 2333-2365, 2010.

[34] J.-M. Coron, "Global asymptotic stabilization for controllable systems without drift," Mathematics of Control, Signals, and Systems, vol. 5, no. 3, pp. 295-312, 1992. 


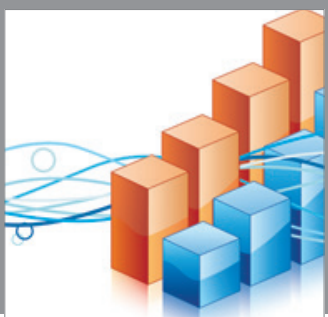

Advances in

Operations Research

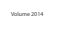

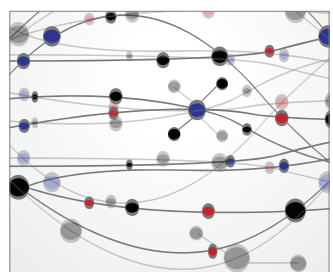

\section{The Scientific} World Journal
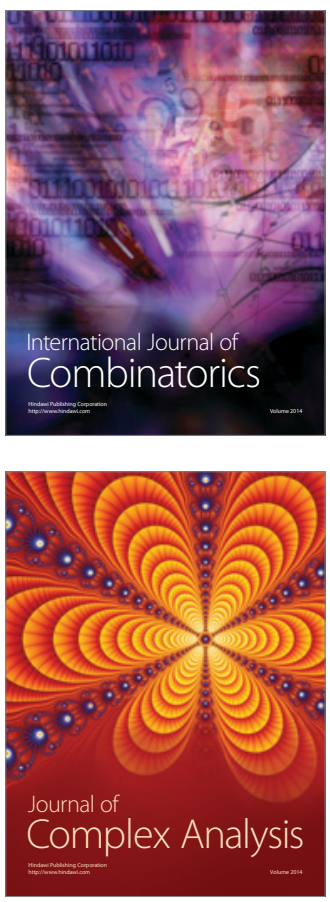

International Journal of

Mathematics and

Mathematical

Sciences
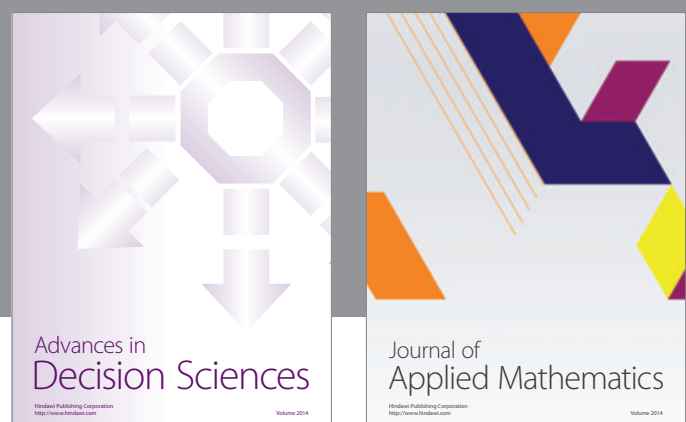

Journal of

Applied Mathematics
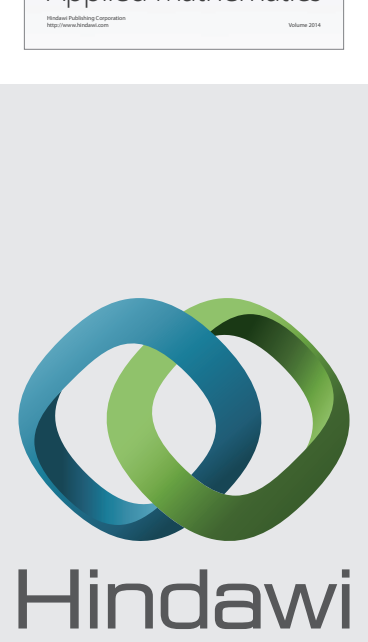

Submit your manuscripts at http://www.hindawi.com
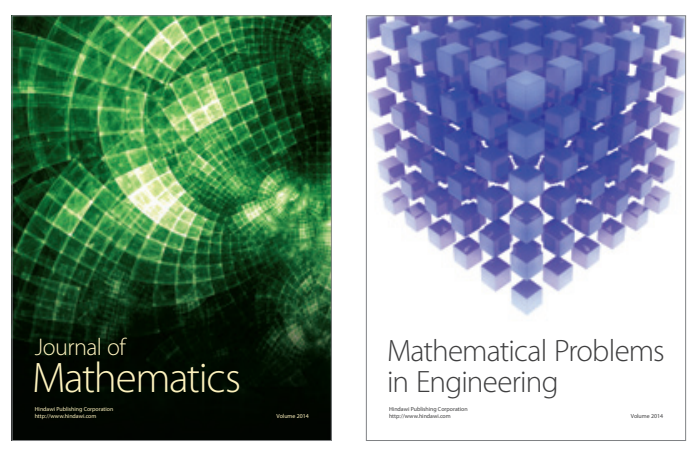

Mathematical Problems in Engineering
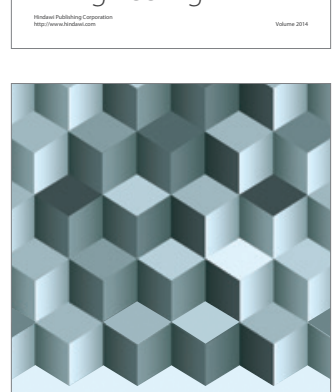

Journal of

Function Spaces
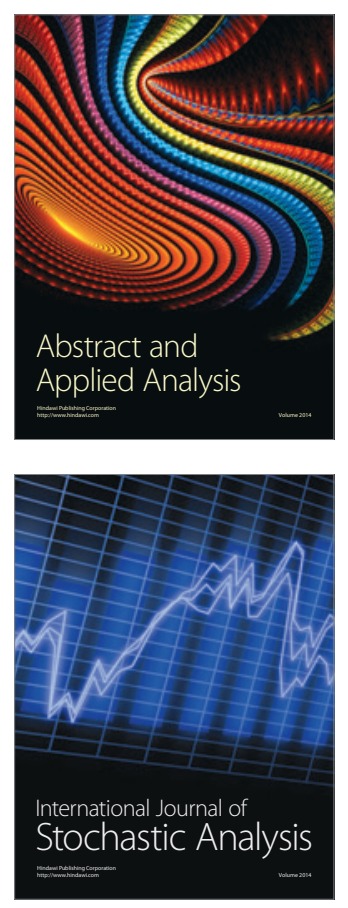

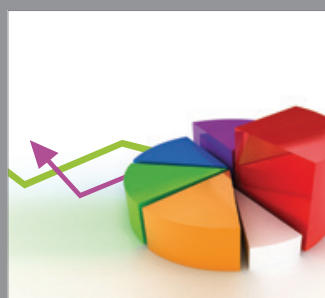

ournal of

Probability and Statistics

Promensencen
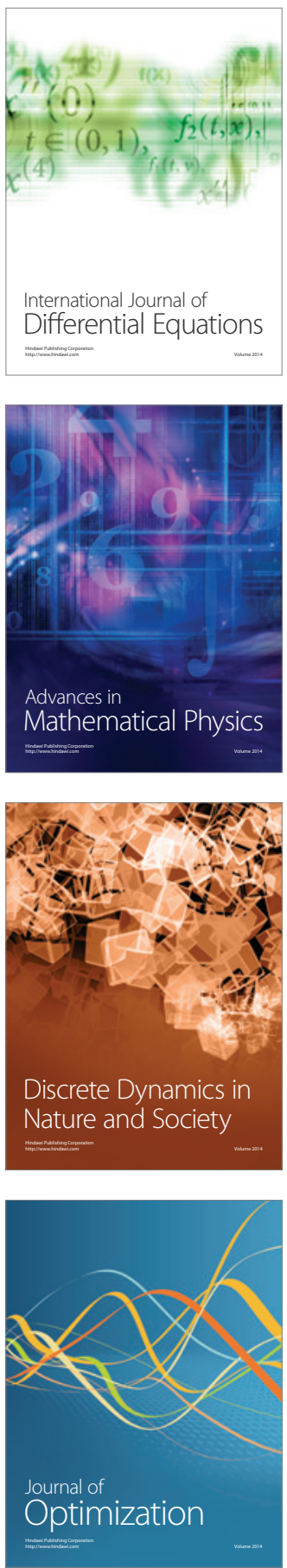